J. Dairy Sci. 91:4542-4551

doi:10.3168/jds.2007-0907

(c) American Dairy Science Association, 2008.

\title{
Molecular Epidemiology and Population Structure of Bovine Streptococcus uberis
}

\author{
M. G. Rato, ${ }^{*}$ R. Bexiga,† S. F. Nunes, $†$ L. M. Cavaco, † C. L. Vilela, $†$ and I. Santos-Sanches ${ }^{\star 1}$ \\ ${ }^{*}$ Centro de Recursos Microbiológicos (CREM), Faculdade de Ciências e Tecnologia, Universidade Nova de Lisboa, Caparica, Portugal \\ †Centro de Investigação Interdisciplinar em Sanidade Animal (CIISA), Faculdade de Medicina Veterinária, Universidade Técnica de Lisboa, \\ Lisbon, Portugal
}

\section{ABSTRACT}

The molecular epidemiology and population structure of 30 bovine subclinical mastitis field isolates of Streptococcus uberis, collected from 6 Portuguese herds (among 12 farms screened) during 2002 and 2003, were examined by using pulsed-field gel electrophoresis (PFGE) for clustering of the isolates and multilocus sequence typing (MLST) to assess the relationship between PFGE patterns and to identify genetic lineages. The 30 isolates were clustered into 18 PFGE types, using a similarity cutoff of $80 \%$, and 3 PFGE types accounted for almost half of the isolates $(46.6 \%)$. These major types were herd specific, suggesting either cowto-cow transmission or infection with isolates from the same environmental reservoirs. The remaining unrelated PFGE types of isolates were from different herds strongly suggesting environmental sources of Strep. uberis infection. All 30 isolates were analyzed by MLST and clustered into 14 sequence types (ST). These ST were found to be novel, either with 10 new alleles of 6 housekeeping genes or with different combinations of previously assigned alleles. Five of these ST were clustered into 3 clonal complexes (lineages), ST-143, ST-86, and ST-5, known to include bovine isolates from several geographic locations (Australia, New Zealand, United Kingdom, Sweden, and Denmark) and 9 singletons. To our knowledge, this is the first report that documents molecular typing studies of bovine isolates of Strep. uberis from Portugal, which were shown to represent novel genomic backgrounds of this pathogen.

Key words: bovine mastitis, Streptococcus uberis, pulsed-field gel electrophoresis, multilocus sequence typing

Received December 1, 2007.

Accepted August 3, 2008.

${ }^{1}$ Corresponding author: isanches@fct.unl.pt

\section{INTRODUCTION}

Bovine mastitis remains a major cause of economic loss in the dairy industry worldwide, despite the widespread implementation of mastitis control strategies. Economic losses are caused mainly by decreased milk production, early culling and death of the animals (Seegers et al., 2003), and increased antibiotic therapy (Erskine et al., 2003).

Contagious mastitis pathogens (such as Streptococcus agalactiae and Staphylococcus aureus) have been brought under control in dairy herds through use of management practices (Bramley and Dodd, 1984; Bradley, 2002). However, mastitis pathogens traditionally classified as environmental (e.g., Streptococcus uberis) are not well controlled by these methods and are, in several countries, the most frequent cause of subclinical and clinical mastitis in both lactating and nonlactating cows, particularly on well-managed farms (Bradley, 2002). Mastitis due to Strep. uberis infection was generally considered the result of environmental exposure to this pathogen (Lopez-Benavides et al., 2007); however, cases of cow-to-cow transmission of Strep. uberis have been reported (Douglas et al., 2000; Phuektes et al., 2001; Zadoks et al., 2003).

Methods based on DNA have been successfully used in epidemiological studies aiming to evaluate strain-specific transmission and to improve infection control measures (Khan et al., 2003; Wieliczko et al., 2002; Zadoks et al., 2003). Pulsed-field gel electrophoresis (PFGE) is the most discriminatory method and has been used for typing a broad range of pathogens including bovine (Baseggio et al., 1997; Wang et al., 1999; Douglas et al., 2000) and human pathogens, being most useful in outbreak investigations (Tenover et al., 1995; Van Belkum et al., 2007). Multilocus sequence typing (MLST) is a valuable tool that allows further investigation of the population structure and evolution of several pathogens (Maiden et al., 1998; http://www.mlst.net/), including Strep. uberis (Pullinger et al., 2006, 2007). For Strep. uberis, 2 MLST schemes have been described, one based on sequencing of 6 loci that include virulence genes and housekeeping genes (Zadoks et al., 2005) and the other 
based on the sequencing of 7 housekeeping genes (Coffey et al., 2006; http://pubmlst.org/suberis/).

In this study we used PFGE and MLST (using the latter scheme referred to above) to characterize a collection of field isolates of Strep. uberis from subclinical bovine mastitis occurring in Portuguese herds during 2002 and 2003 and to document the molecular epidemiology and population structure of the isolates, which would be useful in providing further insights into the evolutionary and population genetics of this pathogen.

\section{MATERIALS AND METHODS}

\section{Strep. uberis Collection}

A total of 30 Strep. uberis field isolates causing bovine subclinical mastitis were included in this study. This collection comprised all Strep. uberis isolates (with the exception of 4 isolates that were lost during storage) recovered from February 2002 to March 2003, from 261 bovine milk samples of 215 animals with subclinical mastitis from 6 dairy farms (of 12 screened) located in the southwestern region of Portugal (Bexiga et al., 2005). The original study (Bexiga et al., 2005) involved milk sampling of 459 quarters from 377 animals from 12 dairy herds. Selection criteria for the herds included in the study were geographical area and willingness of the farmers to participate in the study. The 12 herds enrolled in the study had a mean size of 248 lactating cows, a mean bulk tank SCC of 462,000 cells $/ \mathrm{mL}$, and a mean milk yield at $305 \mathrm{~d}$ of $7,987 \mathrm{~kg}$ of milk. Farms were all zero-grazed, which is typical of continental Portuguese herds. The program WinEpiscope 2.0 (Computer-aided Learning In Veterinary Education, CLIVE, University of Edinburgh, UK ) was used to calculate the number of cows for milk sampling in each farm. Briefly, a representative number of animals (for an expected prevalence of $50 \%$ of subclinical mastitis at cow level, with a margin of error of $10 \%$ and a confidence level of 95\%) was submitted to a California Mastitis Test, with quarters presenting a score of 3 being chosen for quarter sampling (score 3 on the California Mastitis Test, used to define subclinical mastitis on a scale of 0 to 3 , with 0 being negative and 3 being strong positive). Quarters were only sampled once during the study period. Different sampling visits distant in time on the same farm depended on the calculated number of animals to sample.

In total, 351 of 459 samples were positive for bacterial growth. Of those, Strep. uberis and Staph. aureus were found in $10 \%$ of the samples, Strep. agalactiae in $18.2 \%$, and CNS in $34.5 \%$ (Bexiga et al., 2005).

The distribution of the 30 Strep. uberis isolates on the 6 farms (coded F, K, C, D, I, and J) was as follows: 18 isolates from farm F, 6 isolates from farm I, 3 isolates from farm $\mathrm{K}$, and single isolates from farms $\mathrm{C}, \mathrm{D}$, and J. All isolates were collected from different animals. Detailed information about the 30 isolates, including source, date of isolation, and, where appropriate, the identification number, breed, and disease status of the cow is available on the Strep. uberis isolates MLST database (http://pubmlst.org/suberis/).

\section{Strep. uberis Identification}

All isolates were cultured in sheep blood agar media and were identified as Strep. uberis using the API-20 STREP (bioMérieux, Marcy l'Etoile, France) and BBL Crystal Gram-Positive (Becton Dickinson and Co., Franklin Lakes, NJ) identification systems. Differentiation between Strep. uberis and Strep. parauberis, which are phenotypically indistinguishable (Facklam, 2002), was performed by PCR amplification of the $16 \mathrm{~S}$ ribosomal DNA (rDNA) gene of all the isolates, using generic primers for gram-positive bacteria (Takahashi et al., 1997) and sequencing with the same primers used for amplification. Automatic sequencing was carried out by Macrogen (Seoul, Korea) or by STAB-Vida (Lisbon, Portugal). Sequence data were edited using SeqMan, DNAstar (DNAstar Inc., Madison, WI). Each of the sequences of the $16 \mathrm{~S}$ rDNA gene of the 30 isolates was compared with the sequence of the $16 \mathrm{~S}$ rDNA gene of Strep. uberis reference strain HN1 (GenBank accession no. AB023576) and then compared with the sequences of all the species deposited in the GenBank database using BlastN (http://www.ncbi.nlm.nih.gov/ BLAST/).

\section{PFGE Typing and Cluster Analysis}

The 30 Strep. uberis isolates were analyzed by PFGE as described previously (Chung et al., 2000). Briefly, a $6-\mathrm{mL}$ culture of cells in early stationary phase was harvested and washed in $1 \mathrm{~mL}$ of Tris-NaCl buffer (10 $\mathrm{m} M$ Tris, $\mathrm{pH} 8 ; 1 \mathrm{M} \mathrm{NaCl}$ ), and resuspended in $200 \mu \mathrm{L}$ of the same buffer. Concentrations were adjusted to an optical density of 5.0 at $620 \mathrm{~nm}$. This cell suspension was then diluted 1:1 with $150 \mu \mathrm{L}$ of $1.5 \%$ low-gellingtemperature agarose (SeaPlaque, FMC Bioproducts, Rockland, ME) in the same Tris- $\mathrm{NaCl}$ buffer; disks of $20 \mu \mathrm{L}$ were allowed to solidify for $5 \mathrm{~min}$ at $-20^{\circ} \mathrm{C}$. The cells were lysed by incubation of the disks at $37^{\circ} \mathrm{C}$ for 5 $\mathrm{h}$ with $50 \mu \mathrm{g}$ of RNase I, $1 \mathrm{mg}$ of lysozyme, and $5 \mathrm{U}$ of mutanolysine in $1 \mathrm{~mL}$ of lysis buffer $(6 \mathrm{~m} M$ Tris, $\mathrm{pH} 8$, $1 M \mathrm{NaCl}, 0.1 \mathrm{M}$ EDTA, pH 8, $0.2 \%$ deoxycholate, $0.5 \%$ sarkosyl, $0.5 \%$ Brij 58 ). The disks were next incubated in a solution of proteinase $\mathrm{K}(1 \mathrm{mg} / \mathrm{mL})$ in EDTAsarkosyl buffer (0.5 $M$ EDTA, pH 9, 1\% Sarkosyl) at 
A

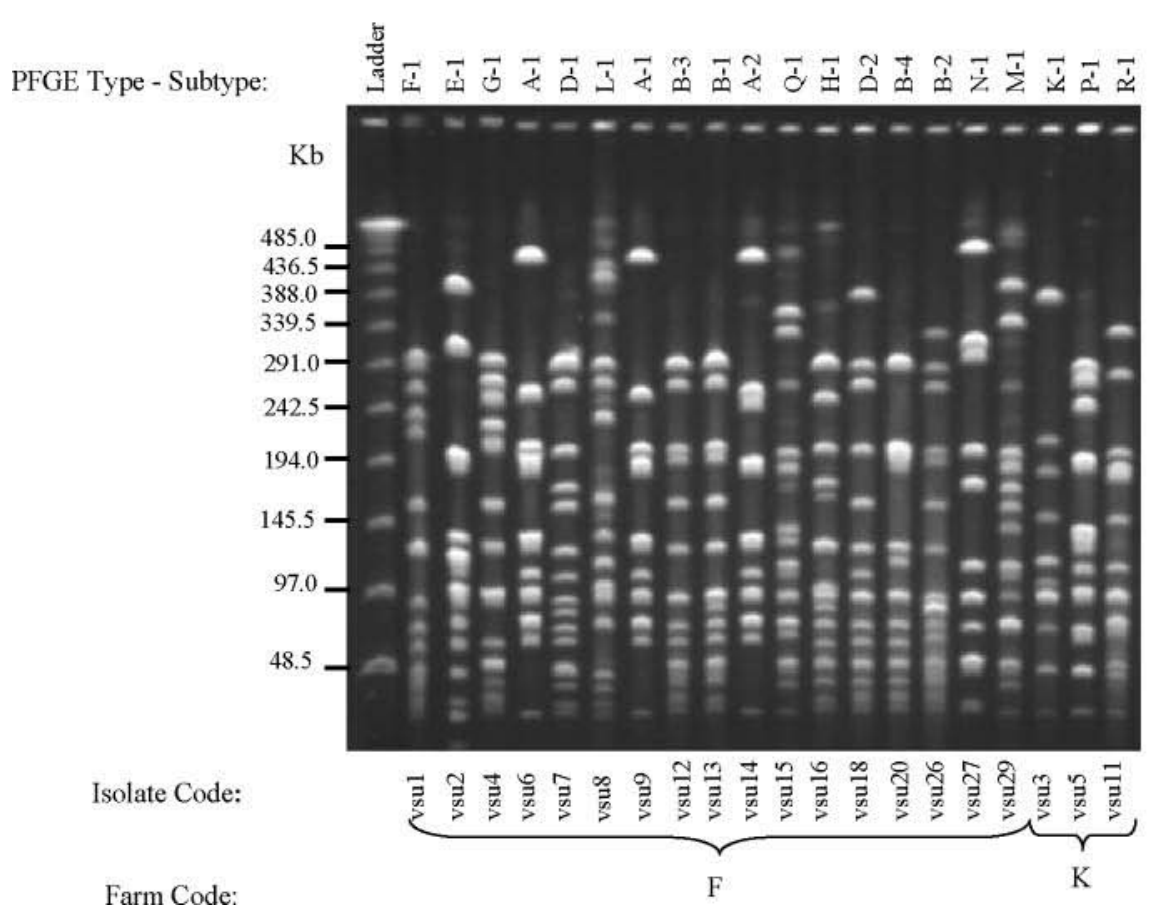

B

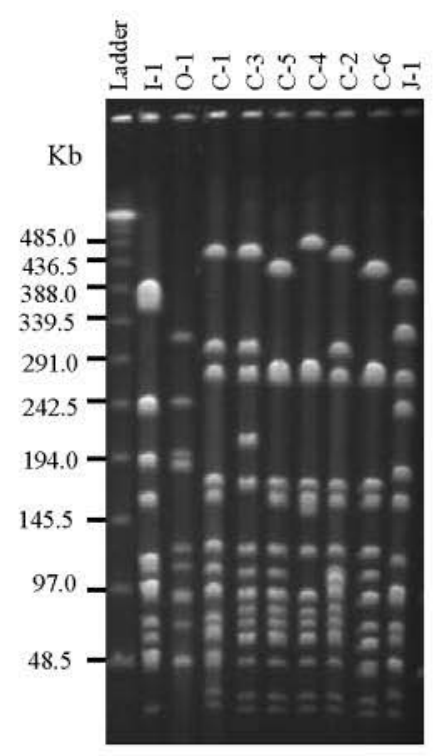

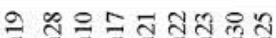

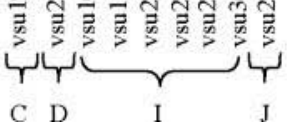

C

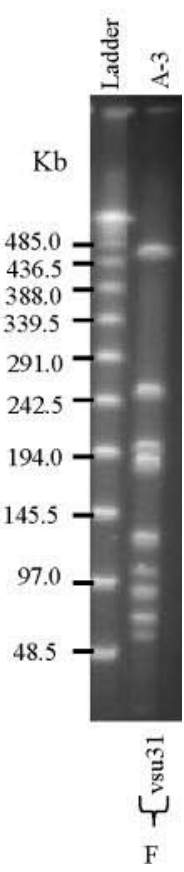

Figure 1. Pulsed-field gel electrophoresis (PFGE) profiles of SmaI-digested genomic DNA from Streptococcus uberis subclinical mastitis isolates collected at 6 Portuguese dairy herds. Ladder = Lambda PFGE ladder marker (New England BioLabs, Beverly, MA). A) PFGE typesubtype patterns of isolates from farm F and K; B) PFGE type-subtype patterns of isolates from farms C, D, I, and J; C) PFGE type-subtype patterns of 1 isolate from farm $\mathrm{F}$.

$50^{\circ} \mathrm{C}$ for $18 \mathrm{~h}$. The agarose disks were washed 8 times in $13 \mathrm{~mL}$ of Tris-EDTA buffer $(10 \mathrm{~m} M$ Tris, $1 \mathrm{~m} M$ EDTA, pH 7.5) for 30 min with gentle agitation. The DNA was then considered purified and was stored in approximately $1 \mathrm{~mL}$ of Tris-EDTA buffer at $4^{\circ} \mathrm{C}$. The DNA was digested with $20 \mathrm{U}$ of $S m a \mathrm{I}$ (New England BioLabs, Beverly, MA), and PFGE was performed in a CHEF DR-III (contour-clamped homogeneous electric field) apparatus (Bio-Rad, Hercules, CA) for $23 \mathrm{~h}$. The running parameters were as follows: initial pulse $=5$ $\mathrm{s}$; final pulse $=35 \mathrm{~s}$; voltage $=200 \mathrm{~V}$; temperature $=$ $11.3^{\circ} \mathrm{C}$. Ethidium bromide $(0.5 \mu \mathrm{g} / \mathrm{mL})$ was used to stain the gels. Images were captured using the Gel Doc XR system and the Quantity One 1-D Analysis Software (Bio-Rad). The PFGE lambda marker (New England Biolabs) was used as a molecular weight standard.

All PFGE profiles or patterns were analyzed visually and by computer-assisted cluster analysis using the software BioNumerics v. 4.0 (Applied Maths, SintMartens-Latem, Belgium). Levels of similarity between fingerprints were calculated by using Dice coefficient, and an unweighted pair group method using arithmetic averages (UPGMA) was used for clustering to produce band-based dendrograms (with a band position tolerance of $1.5 \%$ and no optimization). Groups of patterns with no observed band differences (corresponding to a level of similarity of $100 \%$ ) were considered indistinguishable and were assigned to the same subtype of a PFGE type. Patterns with variation up to 6 bands were considered related according to previous suggested criteria (Tenover et al., 1995) and were clustered in most cases above $80 \%$ similarity. These were assigned to different subtypes of a PFGE type. Patterns with variation of 6 or more bands (corresponding to levels of similarity of less than $80 \%$ ) were not considered related and were assigned to distinct PFGE types. All PFGE types were designated with uppercase letters and each of their subtypes (subclonal variants) was identified in addition by a numeral suffix.

\section{MLST and eBURST Analysis}

All isolates were analyzed by MLST. The DNA was extracted as described for Group A Streptococcus at http://www.cdc.gov/ncidod/biotech/strep/proto-

col_emm-type.htm. The primers used were described previously (www.pubmlst.org/suberis/info/protocol. shtml; Coffey et al., 2006). The PCR amplification reactions were adapted from the method available at the Strep. uberis MLST Web site (www.pubmlst. 
org/suberis/info/protocol.shtml). A $50-\mu \mathrm{L}$ reaction included $1.25 \mathrm{U}$ of Taq polymerase (MBI Fermentas, Vilnius, Lithuania), $30 \mathrm{pmol}$ of each primer, $1.5 \mathrm{mM}$ $\mathrm{MgCl}_{2}$ (MBI Fermentas), $200 \mu M$ of deoxynucleoside triphosphate mix (MBI Fermentas), and $2 \mu \mathrm{L}$ of DNA. The PCR amplicons were purified using the Wizard PCR Preps DNA Purification System (Promega, Madison, WI), and sequencing was performed as described above. The sequences were entered at the Strep. $u b$ eris MLST database (www.pubmlst.org/suberis/info/ protocol.shtml) and novel alleles were assigned new numerals. The allelic profile and sequence type (ST) of each isolate are available at the Strep. uberis MLST database (www.pubmlst.org/suberis/info/protocol.sht$\mathrm{ml}$ ). The predicted evolutionary descent of the isolates was evaluated by using e-BURST (http://eburst.mlst. net/) of Strep. uberis data set (eBURSTv3 - Streptococcus uberis), which included 1 example of each ST assigned at the Strep. uberis MLST database. This tool produces a diagram displaying the way each clonal complex may have emerged and diversified from the predicted founding genotype. This diagram is centered on the predicted founding genotype, and the branches represent the predicted descent to the other genotypes in the group. The eBURST uses lines to show radial links from the founder to each of its single locus variant (initial diversification). Only single locus variant links are shown in an eBURST diagram. All isolates that are directly linked on an e-BURST diagram will therefore differ at only a single locus, and the cluster of linked ST represents a clonal complex.

\section{RESULTS}

\section{S rDNA Gene Sequence Analysis for Strep. uberis Identification}

Comparative analysis with the $16 \mathrm{~S}$ rDNA gene sequence of the Strep. uberis reference strain HN1 from the GenBank database showed that out of the 30 isolates, 13 had maximum identity of 100\%, 16 isolates differed by $1 \mathrm{bp}$, corresponding to a maximum identity of $99 \%$, and 1 isolate differed by $3 \mathrm{bp}$, which also corresponded to a maximum identity of $99 \%$. These variations have been described as microheterogeneity in the 16S rDNA gene sequence within the same species or intraspecies variation of the $16 \mathrm{~S}$ rDNA gene sequence (Clarridge, 2004). Moreover, sequences of the 16S rDNA gene of streptococcal species other than Strep. uberis (including Strep. parauberis) deposited in the GenBank database were $<99 \%$ identical to the $16 \mathrm{~S}$ rDNA gene sequences of the Strep. uberis isolates from the present work. Therefore, we have considered all the isolates as
Strep. uberis taking into account the phenotype and this genotypic tool used for identification.

\section{PFGE Profiles}

Patterns obtained by PFGE ranged from 9 to 15 well-resolved fragments of approximately 10 to $485 \mathrm{~kb}$ (Figure 1, A to C). Percentage similarity among patterns was determined by cluster analysis in a dendrogram (Figure 2).

Epidemiologically plausible groups (clusters) were identified using the cut-off of at least $80 \%$ similarity as described above. Three major clusters (named I, II, and III) included more than 2 isolates at $80 \%$ or greater similarity levels (Figure 2). Cluster I included 4 isolates from farm F. Two isolates (vsu6 and vsu9, collected within a 4-mo interval from 2 cows) had indistinguishable patterns (no observed band differences; see Figure 1) corresponding to a level of similarity of $100 \%$ and were assigned to a same PFGE subtype (A-1) of PFGE type A. The 2 remaining patterns differed by up to 3 bands between isolate pairs and were assigned to different PFGE subtypes (A-2, A-3). The 3 subtypes have $83.3 \%$ similarity. Cluster II included 4 isolates also from farm $\mathrm{F}$, whose patterns differed by 1 to 6 bands between isolate pairs and were assigned to different PFGE subtypes (B-1 to B-4) of PFGE type B with similarity levels ranging from 96.3 to $80.1 \%$. Cluster III included 6 isolates from farm I, whose patterns differed by 1 to 5 bands between isolate pairs and were assigned to different PFGE subtypes (C-1 to C-6) of PFGE type $\mathrm{C}$ with similarity levels ranging from 96.0 to $81.6 \%$. A minor cluster comprised 2 other isolates from farm $\mathrm{F}$ whose patterns differed by 3 bands and were assigned to different PFGE subtypes (D-1, D-2) of PFGE type D with $82.8 \%$ similarity. The remaining isolates were not clustered and were assigned to unique PFGE subtypes.

\section{Allelic Profiles and Sequence Types of Portuguese Strep. uberis Isolates}

The sequences of the 7 gene fragments used for the MLST scheme were determined for all 30 isolates confirmed to be Strep. uberis. These isolates were clustered into $14 \mathrm{ST}$, all found for the first time in this study. These ST were novel because they had different combinations of previously assigned alleles (ST-267, ST-285, ST-286, ST-287, ST-289, and ST-290) or contained 10 new alleles of 6 housekeeping genes (ST-265, ST-266, ST-268, ST-269, ST-270, ST-284, ST-288, and ST-291; Table 1). Within the same cluster (I, II, or III), isolates had the same ST: cluster I included 4 isolates of ST265 , cluster II included 4 isolates of ST-268, and cluster 


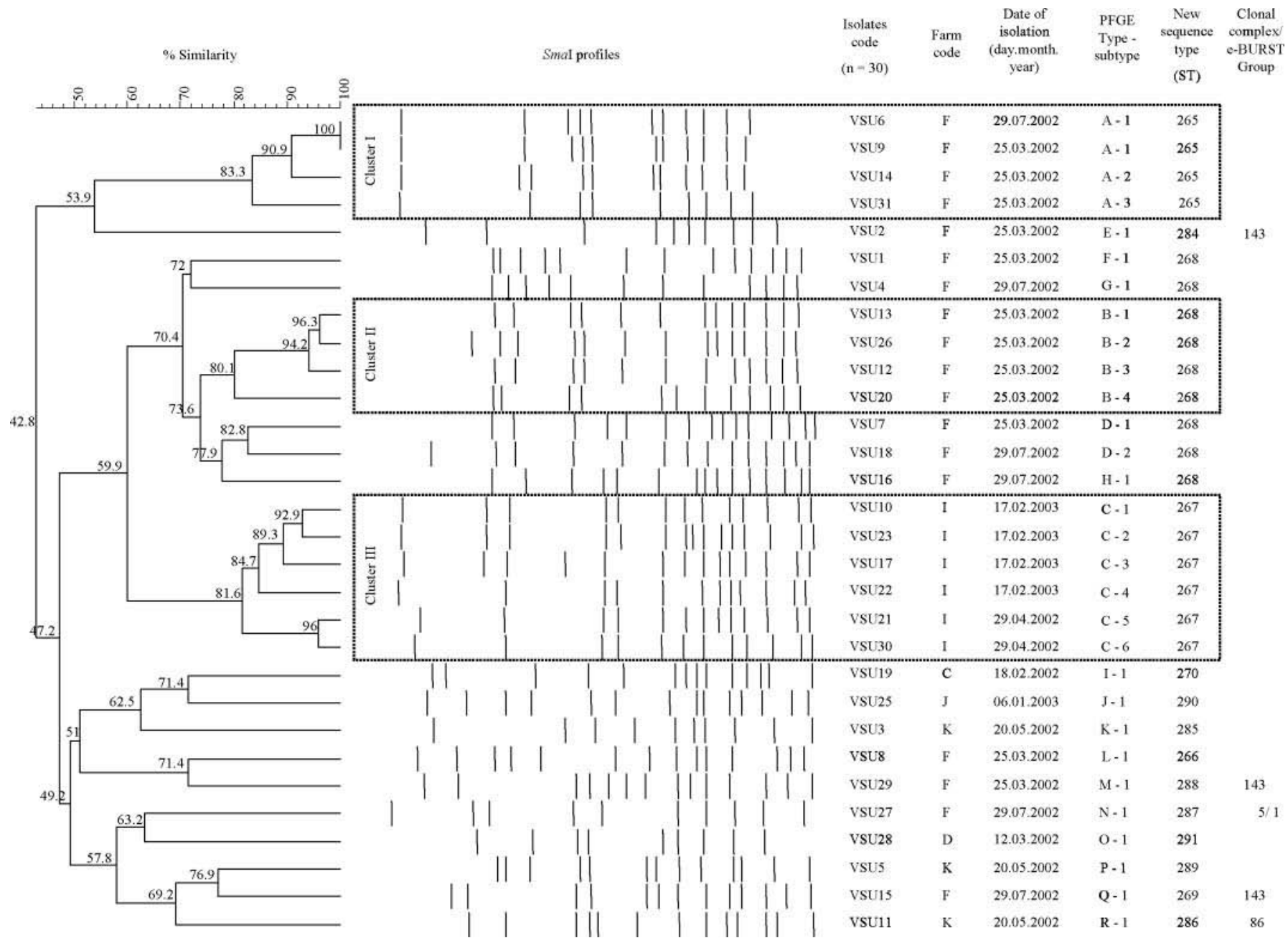

Figure 2. Dendrogram of the pulsed-field gel electrophoresis (PFGE) profiles of Streptococcus uberis subclinical mastitis isolates collected at 6 Portuguese dairy herds, novel multilocus sequence types (ST) and clonal complexes. Isolate code, farm code, and date of isolation of each strain are also represented in the dendrogram. The dendrogram was produced by using Dice coefficients and an unweighted pair group method using arithmetic averages (UPGMA). Default clustering settings of $0.00 \%$ optimization (i.e., the relative distance an entire lane is allowed to shift in matching attempts) and $1.5 \%$ band position tolerance were used.

III included 6 isolates of ST-267 (Figure 2). Some of the PFGE subtypes with $<80 \%$ similarity shared the same ST: PFGE subtypes F-1, G-1, and PFGE subtypes B-1 to B-4 of cluster II with $70.4 \%$ of similarity shared the same ST (ST-268); PFGE subtype H-1 and PFGE subtypes D-1 to D-2 with $77.9 \%$ of similarity shared the same ST (ST-268; Figure 2).

The 18 isolates of farm F were of 7 ST (ST-265, ST266, ST-268, ST-269, ST-284, ST-287, and ST-288) and 11 PFGE types. The 6 isolates of farm I were of a single ST (ST-267) and a unique PFGE type (with 6 subtypes). The 3 isolates of farm $\mathrm{K}$ were of $3 \mathrm{ST}$ (ST285, ST-286, and ST-289) and of 3 unrelated PFGE types. The single isolates from farms C, J, and D were of ST-270, ST-290, and ST-291, respectively, and of 3 unrelated PFGE types (Table 1).
Three clonal complexes or genetic lineages were assigned primarily by the Strep. uberis MLST database curator and all included isolates of single PFGE subtypes. Lineage ST-143 included 3 ST (ST-284, ST-288, and ST-269) of 3 isolates from farm F (vsu2, vsu29, and vsu15) and additionally 122 Strep. uberis strains from dairy herds in Australia, New Zealand, and the UK; lineage ST-5 included the ST-287 of another isolate from farm F (vsu27) and also of 170 Strep. uberis strains from dairy herds in Australia, Denmark, New Zealand, Sweden, and the UK; lineage ST-86 included ST-286 of 1 isolate from farm K (vsu11) and of 41 other Strep. uberis strains from dairy herds in Australia, New Zealand, Sweden, and the UK (data from pubmlst. org/suberis). The remaining 9 ST from this study were singletons. 
Table 1. Allelic profiles and clonal complexes of Streptococcus uberis isolates of bovine subclinical mastitis from Portugal assigned by multilocus sequence typing (MLST)

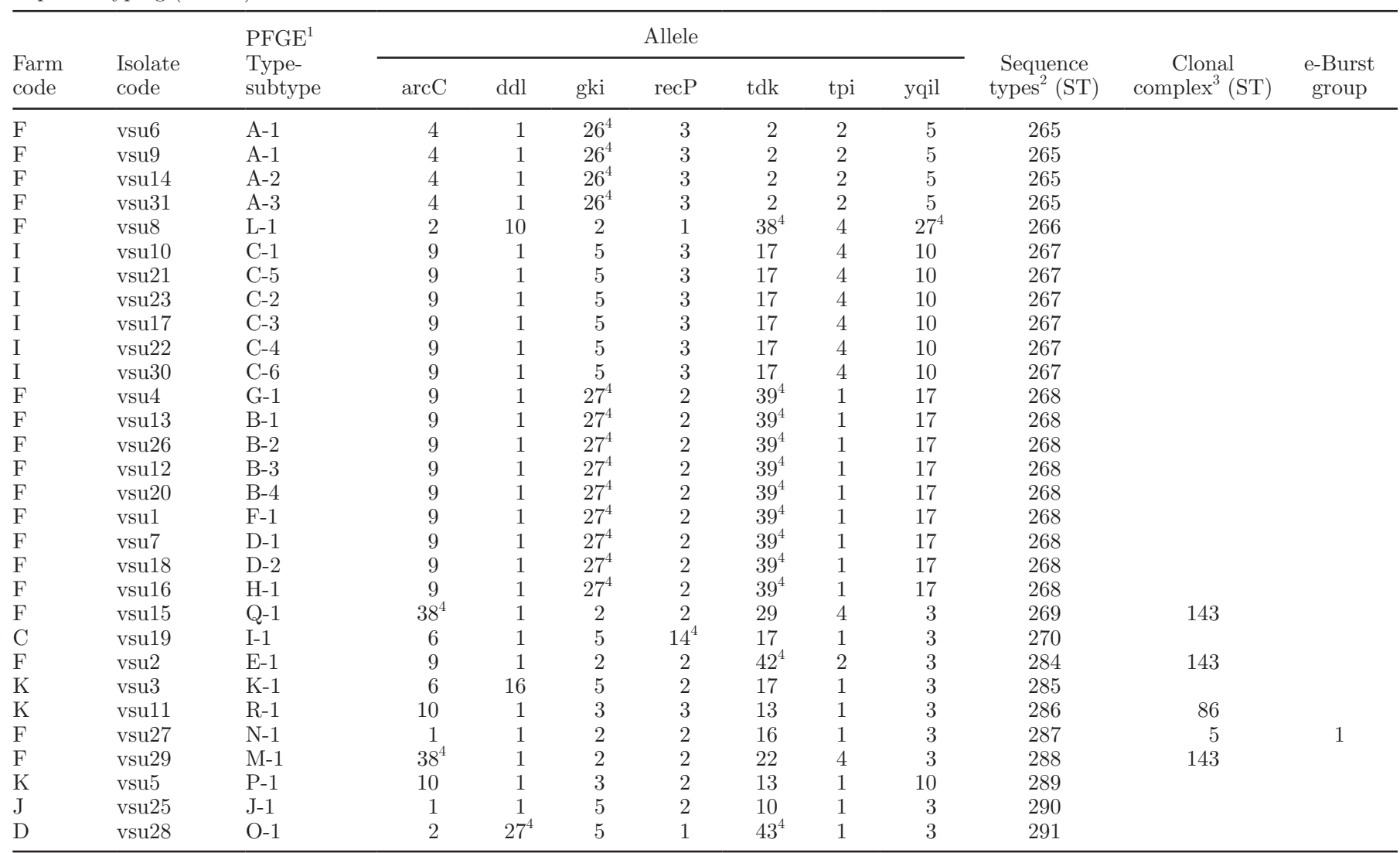

${ }^{1} \mathrm{PFGE}=$ pulsed-field gel electrophoresis.

${ }^{2}$ Novel allelic profiles or sequence types (ST).

${ }^{3}$ MLST clonal complexes (clusters of linked ST) assigned by the Strep. uberis MLST database curator (www.pubmlst.org/suberis/).

${ }^{4}$ Novel allele.

The e-BURST analysis revealed that only ST-287 from this study of lineage ST-5 was included in a clonal complex described as group 1 by eBURSTv3 - Streptococcus uberis software (http://eburst.mlst.net/; Figure 3); ST-287 was found to be a single locus variant of the predicted founder (ST-184) of this major clonal complex (Figure 3), which includes 107 ST of the Strep. uberis MLST database.

\section{DISCUSSION}

Streptococcus uberis is one of the most prevalent pathogens in dairy herds and is considered one of the principal causative agents of bovine mastitis, a very costly disease in dairy industry (Leigh, 1999; Shim et al., 2004). It has been noted that the relative importance of transmission mechanisms and control measures differs between herds and geographical areas (LeBlanc et al., 2006). However, studies on the molecular characterization of field Strep. uberis isolates from Portugal are still not documented and are of utmost importance for a more efficient control of bovine mastitis caused by this pathogen.

This species, together with the contagious pathogen Staph. aureus, accounted for $10 \%$ of all milk samples with positive growth, Strep. agalactiae accounted for $18.2 \%$, and CNS accounted for 34.5\%, during 2002 and 2003, from a study on bovine subclinical mastitis among 12 commercial dairy farms in a particular region of Portugal (Bexiga et al., 2005). Greater prevalence rates of Strep. uberis have been reported in other countries such as New Zealand (Douglas et al., 2000) because the dairy industry there is mainly pasture based. However, continental Portuguese herds are typically zero-grazed, which could explain lower rates of Strep. uberis infection. Moreover, among the 12 commercial dairy farms screened, Strep. uberis was found on only 6 farms (50\%).

Our aim was to assess if subclinical bovine mastitis episodes were associated with multiple PFGE types or 


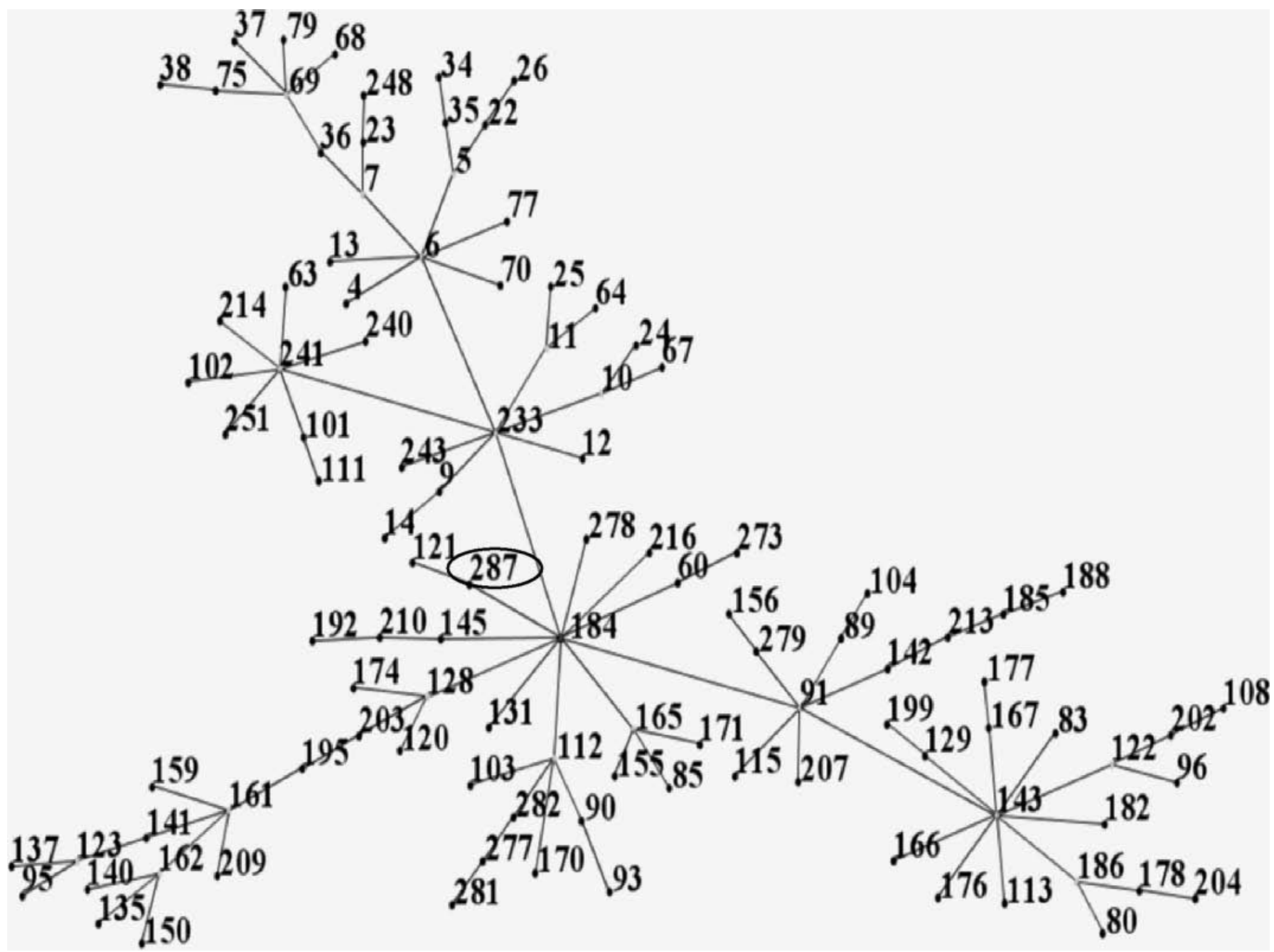

Figure 3. e-BURST diagram determined by eBURSTv3 software (http://eburst.mlst.net/) using 1 example of each sequence type (ST) assigned in the Streptococcus uberis multilocus sequence typing (MLST) database (http://pubmlst.org/suberis/info/protocol.shtml). Only ST-287 (circled) from the present work was assigned to the major clonal complex described as group 1, which includes 107 ST of Strep. uberis MLST database; ST-287 is a single locus variant of the predicted founder (ST-184).

with a limited number of dominant types, which is important information for the implementation of targeted mastitis control programs. Additionally, we aimed to document the genotypic properties of this species in a region not yet surveyed, contributing to increased knowledge about the evolutionary and population genetics of this pathogen by using MLST.

The combined use of the molecular typing techniques mentioned above was a valuable approach to achieve our aims. The prevalence of Strep. uberis varied between herds. The totality of the Strep. uberis isolated in the survey by Bexiga et al. (2005) were included in the present study. Close to half $(44.4 \% ; \mathrm{n}=8 / 18)$ of the Strep. uberis isolates from farm F $(\mathrm{n}=18)$ were clustered into 2 large homogeneous PFGE groups, clusters I and II. Moreover, all the Strep. uberis isolates from farm I $(\mathrm{n}=6)$ were clustered in 1 PFGE homogeneous group (cluster III).

The finding of a limited number of dominant PFGE clusters or PFGE types spread over farms $\mathrm{F}$ and I rather than a wide heterogeneity of PFGE types strongly suggested that cows might have been the source of infections or that acquisition from a common environmental source occurred. Despite that, other heterogeneous PFGE types were detected on farm F, which suggests that several environmental sources might have been the source of infections or that the replacement policy on that farm relied more heavily on buying in animals than occurred on other farms.

Related isolates, according to the PFGE data, were seen to share the same ST; however, PFGE and MLST did not correlate in all cases. Isolates of unrelated 
PFGE types F, G, and B ( $70.4 \%$ similarity) were found to share the same ST (ST-268; Figure 2). In addition, $\mathrm{H}$ and D (77.9\% similarity) shared ST-268. This result was expected because PFGE is sensitive to microvariation and, therefore, often has more discriminatory power (Van Belkum et al., 2007) than MLST, which detects variation that accumulates slowly in housekeeping genes (Maiden et al., 1998).

Moreover, different PFGE subtypes of a same type were observed among isolates from different cows within the same herd collected on the same day of sampling. Examples are isolates of PFGE B-1 to B-4 from farm $\mathrm{F}$ or isolates of PFGE C-1 to C-4 from farm I (Figure 2). In contrast, and of interest, was the finding of 2 isolates with indistinguishable PFGE patterns (100\% similarity; PFGE subtype A-1) collected from 2 cows on farm F collected within a 4-mo interval, which strongly suggests that infection of both cows was caused by the same strain, either because of transmission during milking or by close contact with the same environmental reservoir. Clarification of the importance of these environmental reservoirs to the incidence of infection by typing isolates from different sites on the cow and environmental sites will be required in the future. Nevertheless, the observed differences in PFGE banding patterns may be due to genetic changes in persisting strains within these herds.

Prevalence of Strep. uberis clones as well as persistent Strep. uberis strains within the same herd have been reported previously (Phuektes et al., 2001; Zadoks et al., 2003), although most studies found that the point source of infection is uncommon and the main route of transmission is through environmental sources (Khan et al., 2003; Tomita et al., 2008). These disparities in the epidemiology of Strep. uberis may reflect differences among production systems (McDougall et al., 2004).

More variability was expected between related isolates collected at sampling visits distant in time. In fact, different PFGE subtypes of a same type were observed among isolates from different cows within the same herd collected at 4-mo or 10-mo intervals between sampling. Examples are isolates of PFGE A-1 to A-3, D-1 and D-2 from farm F, or isolates of PFGE C-1 to C-4 and isolates C-5 or C-6 from farm I (Figure 2).

As noted above, in most cases, the MLST results agreed with the fingerprinting data and allowed us, in addition, to infer a population structure for our collection of isolates. All of the ST found in this study were novel, either with 10 new alleles of 6 housekeeping genes or with different combinations of previously assigned alleles. Presently, the Strep. uberis profile database consists of 291 profiles. Among these, ST-265 to ST-270 and ST-284 to ST-291 were the ones described in this study. Interestingly, the 2 large homogeneous
PFGE clusters I and II, which grouped almost half $(44.4 \%)$ of the Strep. uberis from farm F were judged as singletons (ST-265 and ST-268) by MLST. On the other hand, the remaining isolates that were dispersed by minor and unique PFGE patterns and grouped into 4 sequence types (ST-269, ST-284, ST-288, and ST287) were clustered into 2 clonal complexes or lineages (ST-5 and ST-143). It was suggested that isolates belonging to clonal complexes ST-5 and ST-143 might possess virulence factors promoting invasion of host tissue, survival in the host environment, evasion of the host immune response, or internalization in the mammary gland (Tomita et al., 2008). Moreover, a link was observed between clonal complexes ST-5 and ST-143 and carriage of the has $A$ virulence gene (Coffey et al., 2006; Pullinger et al., 2006).

It is intriguing why only the singletons from farm $\mathrm{F}$, rather than the major PFGE cluster I and cluster II (also from farm F), were assigned to these virulent clonal complexes, because these strains might have been hypertransmissible among cows, and therefore would be expected to be more virulent. Our data suggest that strains other than those of clonal complexes ST-5 and ST-143 are also hypervirulent strains.

The occurrence of identical ST between Portugal and other countries was not detected. All ST found in the present work are unique and were found for the first time in this collection, which would be expected because information regarding mastitis isolates from Mediterranean countries is lacking in the MLST database. Nevertheless, ST-287 from the present study was found to be a single locus variant of the predicted founder ST-184 of the major group 1 clonal complex (http://eburst.mlst.net); ST-184 includes one strain from clinical mastitis in Australia and strains from environmental sources in New Zealand.

In conclusion, it seems that the contagious route of mastitis infection is still a problem in Portugal. High prevalence of the contagious mastitis pathogens Strep. agalactiae and Staph. aureus was found among the subclinical mastitis milk samples collected during the original study period (Bexiga et al., 2005). Although Strep. uberis is traditionally classified as an environmental pathogen, it may also be transmitted from cow to cow, probably through the milking process. The PFGE analysis in the present study supports this view, as we observed that a limited number of dominant PFGE types spread in farms $\mathrm{F}$ and I (which accounted for $46.6 \%$ of all isolates). Nevertheless, acquisition from a common source may also be occurring: all farms were zero-grazed and, therefore, infection with Strep. uberis from environmental reservoirs such as pastures was not expected. These results may reflect a possible defect in the implementation of mastitis control programs 
on these farms, and better policies for herd management may be needed. Interestingly, presumably more virulent strains (assigned to virulent clonal complexes) were associated with single PFGE types and not with clonal spread. To our knowledge, this is the first report describing genetic relationships among bacterial isolates of bovine Strep. uberis from Portugal, which were shown to represent novel genomic backgrounds and unique genotypes of this pathogen.

\section{ACKNOWLEDGMENTS}

This work was supported by grant project POCTI/ ESP/48407/2002 (Fundação para a Ciência e Tecnologia, Portugal; FEDER), project FCG PROC 60839 (Fundação Calouste Gulbenkian, Portugal), project CREM/3 - Molecular Characterisation of Pathogenic Bacteria (CREM/Faculdade de Ciências e Tecnologia, Universidade Nova de Lisboa, Portugal), and project CIISA/46 - Bovine Mastitis: epidemiology, prophylactic and therapeutic approaches (CIISA/Faculdade de Medicina Veterinária, Universidade Técnica de Lisboa, Portugal). We gratefully acknowledge Rogério Tenreiro, Tânia Tenreiro, and Sandra Chaves (Instituto de Ciência Aplicada e Tecnologia, Lisbon, Portugal), and Dora Rolo (Department of Microbiology, Hospital Universitario de Bellvitge, Barcelona, Spain) for assistance in the establishment of dendrograms and analysis. The authors also acknowledge Dora Rolo and Renato Pires (Center for Microbial Resources - CREM; Faculdade de Ciencias e Tecnologia, Universidade Nova de Lisboa, Caparica, Portugal) for their technical assistance. This publication made use of the Streptococcus uberis MLST Web site (http://pubmlst.org/suberis) developed by Keith Jolley and located at the University of Oxford (Jolley et al., 2004). The development of this site was funded by the Wellcome Trust. We acknowledge Gillian Pullinger, the Strep. uberis database curator, for assignment of new alleles, sequence types, and clonal complexes.

\section{REFERENCES}

Baseggio, N., P. D. Mansell, J. W. Browning, and G. F. Browning. 1997. Strain differentiation of isolates of streptococci from bovine mastitis by pulsed-field gel electrophoresis. Mol. Cell. Probes 11:349-354.

Bexiga, R., L. M. Cavaco, and C. L. Vilela. 2005. Subclinical bovine mastitis in the Ribatejo-Oeste area. Rev. Port. Cienc. Vet. 100:39-44. (In Portuguese)

Bradley, A. 2002. Bovine mastitis: An evolving disease. Vet. J. 164:116-128.

Bramley, A. J., and F. H. Dodd. 1984. Reviews of the progress of dairy science: Mastitis control - progress and prospects. J. Dairy Res. $51: 481-512$.

Chung, M., H. de Lencastre, P. Matthews, A. Tomasz, I. Adamsson, M. Aires de Sousa, T. Camou, C. Cocuzza, A. Corso, I. Couto, A.
Dominguez, M. Gniadkowski, R. Goering, A. Gomes, K. Kikuchi, A. Marchese, R. Mato, O. Melter, D. Oliveira, R. Palacio, R. SáLeão, I. Santos-Sanches, J. H. Song, P. T. Tassios, and P. Villari, and Multilaboratory Project Collaborators. 2000. Molecular typing of methicillin-resistant Staphylococcus aureus by pulsedfield gel electrophoresis: Comparison of results obtained in a multilaboratory effort using identical protocols and MRSA strains. Microb. Drug Resist. 6:189-198.

Clarridge, J. E. 2004. Impact of 16S rRNA gene sequence analysis for identification of bacteria on clinical microbiology and infectious diseases. Clin. Microbiol. Rev. 17:840-862.

Coffey, T. J., G. D. Pullinger, R. Urwin, K. A. Jolley, S. M. Wilson, M. C. Maiden, and J. A. Leigh. 2006. First insights into the evolution of Streptococcus uberis: A multilocus sequence typing scheme that enables investigation of its population biology. Appl. Environ. Microbiol. 72:1420-1428.

Douglas, V. L., S. G. Fenwick, D. U. Pfeiffer, N. B. Williamson, and C. W. Holmes. 2000. Genomic typing of Streptococcus uberis isolates from cases of mastitis, in New Zealand dairy cows, using pulsedfield gel electrophoresis. Vet. Microbiol. 75:27-41.

Erskine, R. J., S. Wagner, and F. J. DeGraves. 2003. Mastitis therapy and pharmacology. Vet. Clin. Food Anim. 19:109-138.

Facklam, R. 2002. What happened to the streptococci: Overview of taxonomic and nomenclature changes. Clin. Microbiol. Rev. 15:613-630.

Jolley, K. A., M. S. Chan, and M. C. Maiden. 2004. mlstdbNetDistributed multi-locus sequence typing (MLST) databases. BMC Bioinformatics 5:86.

Khan, I. U., A. A. Hassan, A. Abdulmawjood, C. Lämmler, W Wolter, and M. Zschöck. 2003. Identification and epidemiological characterization of Streptococcus uberis isolated from bovine mastitis using conventional and molecular methods. J. Vet. Sci. $4: 213-224$.

LeBlanc, S. J., K. D. Lissemore, D. F. Kelton, T. F. Duffield, and K. E. Leslie. 2006. Major advances in disease prevention in dairy cattle. J. Dairy Sci. 89:1267-1279.

Leigh, J. A. 1999. Streptococcus uberis: A permanent barrier to the control of bovine mastitis? Vet. J. 157:225-238.

Lopez-Benavides, M. G., J. H. Williamson, G. D. Pullinger, S. J. LacyHulbert, R. T. Cursons, and J. A. Leigh. 2007. Field observations on the variation of Streptococcus uberis populations in a pasturebased dairy farm. J. Dairy Sci. 90:5558-5566.

Maiden, M. C. J., J. A. Bygraves, E. Feil, G. Morelli, J. E. Russell, R. Urwin, Q. Zhang, J. Zhou, K. Zurth, D. A. Caugant, I. M. Feavers, M. Achtman, and B. G. Spratt. 1998. Multilocus sequence typing: A portable approach to the identification of clones within populations of pathogenic microorganisms. Proc. Natl. Acad. Sci. USA 95:3140-3145.

McDougall, S., T. J. Parkinson, M. Leyland, F. M. Anniss, and S G. Fenwick. 2004. Duration of infection and strain variation in Streptococcus uberis isolated from cows' milk. J. Dairy Sci. 87:2062-2072.

Phuektes, P., P. D. Mansell, R. S. Dyson, N. D. Hooper, J. S. Dick, and G. F. Browning. 2001. Molecular epidemiology of Streptococcus uberis isolates from dairy cows with mastitis. J. Clin. Microbiol. 39:1460-1466.

Pullinger, G. D., T. J. Coffey, M. C. Maiden, and J. A. Leigh. 2007. Multilocus-sequence typing analysis reveals similar populations of Streptococcus uberis are responsible for bovine intramammary infections of short and long duration. Vet. Microbiol. 119:194204.

Pullinger, G. D., M. López-Benavides, T. J. Coffey, J. H. Williamson, R. T. Cursons, E. Summers, J. Lacy-Hulbert, M. C. Maiden, and J. A. Leigh. 2006. Application of Streptococcus uberis multilocus sequence typing: Analysis of the population structure detected among environmental and bovine isolates from New Zealand and the United Kingdom. Appl. Environ. Microbiol. 72:1429-1436.

Seegers, H., C. Fourichon, and F. Beaudeau. 2003. Production effects related to mastitis and mastitis economics in dairy cattle herds. Vet. Res. 34:475-491. 
Shim, E. H., R. D. Shanks, and D. E. Morin. 2004. Milk losses and treatment costs associated with two treatment protocols for clinical mastitis in dairy cows. J. Dairy Sci. 87:2702-2708.

Takahashi, T., M. Kaneko, Y. Mori, M. Tsuji, N. Kikuchi, and T. Hiramune. 1997. Phylogenetic analyses of Staphylococcus based on the 16S rDNA sequence and assignment of clinical isolates from animals. J. Vet. Med. Sci. 59:775-783.

Tenover, F. C., R. D. Arbeit, R. V. Goering, P. A. Mickelsen, B. E. Murray, D. H. Persing, and B. Swaminathan. 1995. Interpreting chromosomal DNA restriction patterns produced by pulsed-field gel electrophoresis: Criteria for bacterial strain typing. J. Clin. Microbiol. 33:2233-2239.

Tomita, T., B. Meehan, N. Wongkattiya, J. Malmo, G. Pullinger, J. Leigh, and M. Deighton. 2008. Identification of Streptococcus uberis multilocus sequence types highly associated with mastitis. Appl. Environ. Microbiol. 74:114-124.

Van Belkum, A., P. T. Tassios, L. Dijkshoorn, S. Haeggman, B. Cookson, N. K. Fry, V. Fussing, J. Green, E. Feil, P. Gerner-Smidt, S. Brisse, and M. Struelens. 2007. Guidelines for the validation and application of typing methods for the use in bacterial epidemiology Clin. Microbiol. Infect. 13(Suppl. 3):1-46.

Wang, S. M., M. A. Deighton, J. A. Capstick, and N. Gerraty. 1999. Epidemiological typing of bovine streptococci by pulsed-field gel electrophoresis. Epidemiol. Infect. 123:317-324.

Wieliczko, R. J., J. H. Williamson, R. T. Cursons, S. J. Lacy-Hulbert, and M. W. Woolford. 2002. Molecular typing of Streptococcus uberis isolates from cases of bovine mastitis. J. Dairy Sci 85:2149-2154.

Zadoks, R. N., B. E. Gillespie, H. W. Barkema, O. C. Sampimom, S. P. Oliver, and Y. H. Schukken. 2003. Clinical, epidemiological and molecular characteristics of Streptococcus uberis infections in dairy herds. Epidemiol. Infect. 130:335-349.

Zadoks, R. N., Y. H. Schukken, and M. Wiedmann. 2005. Multilocus sequence typing of Streptococcus uberis provides sensitive and epidemiologically relevant subtype information and reveals positive selection in the virulence gene pauA. J. Clin. Microbiol. 43:2407-2417. 